\title{
Sleep apnoea is a common occurrence in females
}

\author{
Karl A. Franklin*, Carin Sahlin\# ${ }^{\#}$ Hans Stenlund ${ }^{\star}$ and Eva Lindberg ${ }^{+}$
}

ABSTRACT: Obstructive sleep apnoea (OSA) is primarily regarded as a male disorder, presenting with snoring, daytime sleepiness and cardiovascular disease. We aimed to determine the frequency of sleep apnoea among females in the general population.

We investigated 400 females from a population-based random sample of 10,000 females aged 20-70 yrs. They answered a questionnaire and performed overnight polysomnography.

OSA (apnoea/hypopnoea index $(\mathrm{AHI}) \geqslant 5$ ) was found in $50 \%(95 \% \mathrm{Cl} 45-55 \%)$ of females aged 20-70 yrs. Sleep apnoea was related to age, obesity and hypertension, but not to daytime sleepiness. Severe sleep apnoea $(\mathrm{AHI} \geqslant 30)$ was present in $14 \%(95 \% \mathrm{Cl} 8.1-21 \%)$ of females aged $55-70$ yrs and in $31 \%(95 \% \mathrm{Cl} 12-50 \%)$ of obese females with a body mass index of $\geqslant 30 \mathrm{~kg} \cdot \mathrm{m}^{-2}$ aged 55-70 yrs. Sleep apnoea with daytime sleepiness and sleep apnoea with hypertension were observed as two different phenotypes of OSA.

OSA occurs in $50 \%$ of females aged $20-70$ yrs. $20 \%$ of females have moderate and $6 \%$ severe sleep apnoea. Sleep apnoea in females is related to age, obesity and hypertension, but not to daytime sleepiness. When searching for sleep apnoea in females, females with hypertension or obesity should be investigated.

KEYWORDS: Daytime sleepiness, epidemiology, hypertension, phenotypes, polysomnography, sleep apnoea syndromes, females' health

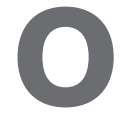
bstructive sleep apnoea (OSA) is primarily regarded as a male disorder. Sufferers snore and experience daytime sleepiness, and run an increased risk of hypertension, stroke and early death [1-9]. Nasal continuous positive airway pressure (CPAP) and oral appliances in the form of mandibular advancement devices during sleep reduce apnoeas, daytime sleepiness and blood pressure [2, 10-14].

A mean of $\geqslant 5$ apnoeas and hypopnoeas per hour of sleep according to overnight polysomnography with continuous respiratory monitoring and sleep staging using electroencephalography is the diagnostic criterion for sleep apnoea [2]. In 1993, YounG et al. [15] reported that $9 \%$ of middle-aged American females had sleep apnoea and that $2 \%$ were also sleepy during the daytime. There are, however, only a few epidemiological studies in females and the frequency of sleep apnoea in females is still uncertain, especially when using modern diagnostic equipment and new scoring recommendations [15-19]. Oronasal thermistors were used in previous prevalence studies and not nasal pressure sensors, which are now recommended when diagnosing sleep apnoea $[2,20]$.
Our knowledge of snoring, daytime sleepiness, hypertension, age and obesity in females with sleep apnoea is also limited.

We aimed to determine the frequency of sleep apnoea among females in the population and associated factors.

\section{METHODS}

\section{Subjects}

In April 2000, a postal questionnaire was sent to 10,000 randomly selected females aged $\geqslant 20$ yrs from the general population in the Municipality of Uppsala, Sweden, in the population-based study entitled "Sleep and Health in Females" [21]. The response rate was $71.6 \%$ for all females. It was $72.9 \%$ in females aged $20-44 \mathrm{yrs}, 74.8 \%$ in females aged $45-54 \mathrm{yrs}, 77.8 \%$ in females aged $55-70$ yrs and $55.0 \%$ in females aged $\geqslant 70$ yrs. The questionnaire included the following question on snoring: "How often do you snore loudly and disturbingly?", with the following five alternative answers: "never", "seldom", "sometimes", "often" and "very often ". Participants reporting loud and disturbing snoring "often" or "very often", which occurred in 7.6\%, were characterised as habitual snorers [22].

\section{AFFILIATIONS}

*Dept of Surgical and Perioperative Sciences, Surgery, Umeå University, ${ }^{\#}$ Dept of Public Health and Clinical Medicine, Medicine, Umeå University,

-Dept of Public Health and Clinical Medicine, Epidemiology and Public Health, Umeå University, Umeå, and ${ }^{+}$Dept of Medical Sciences, Respiratory Medicine and Allergology, Uppsala University, Uppsala, Sweden.

\section{CORRESPONDENCE}

KA. Franklin

Dept of Surgery

Umeå University

SE-901 85 Umeå

Sweden

E-mail: karl.franklin@surgery.umu.se

Received:

Dec 042011

Accepted after revision:

June 102012

First published online:

Aug 162012 
Full-night polysomnographic recordings were obtained in 400 nonpregnant females aged 20-70 yrs from November 2001 to February 2004. Excluded from polysomnography were five females in the snoring group who were treated for sleep apnoea. Females were randomly selected from responders in phase 1, with over-sampling of habitual snorers, and comprised 230 habitually snoring females and 170 females from the complete sample [23, 24]. Another 177 females from the randomly selected group, and 121 females from the snoring group declined polysomnography and they were replaced with other females from each group by random.

The written informed consent of all the participants was obtained and the study protocol was approved by the Ethics Committee at the Medical Faculty at Uppsala University, Uppsala, Sweden.

\section{Procedures}

Overnight ambulatory polysomnography (Embla; Flaga hf, Reykjavik, Iceland) included continuous recordings of electroencephalograms (C3-A2 and C4-A1), electro-oculograms, electromyograms (submental and bilateral anterior tibialis muscles), air flow with a three-port oro-nasal thermistor and a nasal flow pressure sensor, respiratory effort from piezo-electric belts (Resp-EZ; EPM Systems, Midlothian, VA, USA), finger pulse oximetry (Embla A10 flex Sensor), electrocardiograms (V5) and a body position sensor. Sensors were attached by nurses in the evening and the recordings were then performed unattended at home. Data loss occurred in six out of 400 home studies and these females were re-examined within a short period.

All recordings were scored manually (by C. Sahlin). An obstructive apnoea was defined as the cessation of airflow in both nasal pressure and oro-nasal thermistor for at least $10 \mathrm{~s}$ with continuing abdominal and thoracic movements according to the American Academy of Sleep Medicine [2]. An obstructive hypopnoea was defined as a $50 \%$ reduction in both oro-nasal thermistor and nasal pressure for at least $10 \mathrm{~s}$, compared with baseline, accompanied by abdominal and thoracic movements in combination with an arousal or an oxygen desaturation of $\geqslant 3 \%$ [2]. Central apnoeas were scored at the cessation of both oro-nasal thermistor and nasal pressure for $10 \mathrm{~s}$ without respiratory movements. Sleep was scored in 30-s epochs according to RECHTSCHAFFEN and KALES [25]. The obstructive apnoea/hypopnoea index (AHI) was defined as the mean number of obstructive apnoeas and hypopnoeas per hour of sleep. Sleep apnoea was considered when the AHI was $\geqslant 5$. Moderate sleep apnoea was considered when the AHI was 15$<30$ and severe sleep apnoea when the AHI was $\geqslant 30$ [2].

\begin{tabular}{ll}
\hline TABLE 1 & $\begin{array}{l}\text { Weighted mean characteristics of participating } \\
\text { females }\end{array}$ \\
\hline Subjects & Mean or \% (95\% Cl) \\
\hline Age yrs & $48(46-49)$ \\
Body mass index $\mathbf{~ k g} \cdot \mathbf{m}^{-2}$ & $25(25-26)$ \\
Hypertension \% & $15(11-19)$ \\
Current smoking \% & $16(13-30)$ \\
Alcohol dependence \% & $5.6(3.0-8.0)$ \\
\hline
\end{tabular}

Height and weight were measured in the morning after polysomnography. Body mass index (BMI) was defined as the weight in kilograms divided by the square of the height in metres. Systolic and diastolic blood pressure was measured in the supine position after 15 mins' rest during the daytime. Subjects with systolic blood pressure of $>140 \mathrm{mmHg}$ and/or diastolic blood pressure of $>90 \mathrm{mmHg}$ were invited for measurements on two other days and the mean of the measurements was calculated. Hypertension was defined as the ongoing treatment of physician-diagnosed hypertension or systolic blood pressure of $>140 \mathrm{mmHg}$ and/or diastolic blood pressure of $>90 \mathrm{mmHg}$.

A questionnaire was administered during the day before polysomnography. It included questions on snoring, daytime sleepiness, smoking and alcohol. Habitual snoring was considered at a response of "often" or "very often" to the question "How often do you snore loudly and disturbingly?" The alternative responses were "never", "seldom", "sometimes", "often" or "very often". Apnoea witnessed by a bed partner was considered at a response of "sometimes", "often" or "very often".

Daytime sleepiness was scored according to the Epworth Sleepiness Scale (ESS) [26]. Responders are asked to rate their likelihood of dozing in eight different situations on a fourpoint scale. The summary score varies from 0 to 24 . Excessive daytime sleepiness was considered at an ESS score of $\geqslant 10$.

Current smoking was defined as smoking $\geqslant 1$ cigarettes a day. Alcohol dependence was defined using the cut down, annoyed by criticism, guilty about drinking and eye-opener drinks questionnaire (CAGE) [27]. Subjects who answered yes to at least two of the four questions were defined as being alcohol dependent.

\section{Statistical analysis}

Weighting was applied because the individuals were sampled with unequal probability due to the over-sampling of habitually snoring females. Present nonsnoring females and habitually snoring females were weighted to the source population of 6,515 nonsnoring females and 536 habitually snoring females respectively. All the results were weighted to the source population, in order to present data on sleep from females in the general population. The data are presented as weighted means or proportions ( $95 \%$ confidence interval). Univariate and multivariate logistic regression was performed. All calculations were based on weighted data.

\section{TABLE 2 Sleep characteristics}

Weighted mean $(95 \% \mathrm{Cl})$

\begin{tabular}{lc}
\hline Total sleep time min & $392(472-481)$ \\
Stage $\mathbf{1} \%$ of TST & $8(7.8-8.8)$ \\
Stage $\mathbf{2} \%$ of TST & $63(62-63)$ \\
Stage $3-\mathbf{4} \%$ of TST & $10(9.8-11)$ \\
REM \% of TST & $19(18-20)$ \\
\hline
\end{tabular}

TST: total sleep time; REM: rapid eye movement 


\begin{tabular}{|c|c|c|c|c|}
\hline \multirow[t]{3}{*}{ TABLE 3} & \multicolumn{4}{|c|}{$\begin{array}{l}\text { Prevalence in \% of mild sleep apnoea (apnoea/ } \\
\text { hypopnoea index }(\mathrm{AHI}) \geqslant 5) \text {, moderate sleep } \\
\text { apnoea }(\mathrm{AHI} \geqslant 15) \text { and severe sleep apnoea } \\
(\mathrm{AHI} \geqslant 30) \text { in different subgroups of the } \\
\text { population }\end{array}$} \\
\hline & & \multicolumn{3}{|c|}{ AHI } \\
\hline & & $\geqslant 5$ & $\geqslant 15$ & $\geqslant 30$ \\
\hline \multicolumn{5}{|l|}{ Age yrs } \\
\hline \multicolumn{2}{|c|}{ Total aged $20-70$ yrs } & $50(45-55)$ & $20(17-25)$ & $5.9(3.7-8.3)$ \\
\hline \multicolumn{2}{|l|}{$20-44$} & $24(18-31)$ & $4.2(1.0-7.4)$ & $0.1(0.0-0.6)$ \\
\hline \multicolumn{2}{|l|}{$45-54$} & $56(47-65)$ & $17(11-24)$ & $4.6(0.8-8.5)$ \\
\hline \multicolumn{2}{|l|}{$55-70$} & 75 (67-82) & $43(34-52)$ & $14(8.1-21)$ \\
\hline \multicolumn{2}{|l|}{$p$-value } & $<0.001$ & $<0.001$ & 0.008 \\
\hline \multicolumn{5}{|l|}{$\mathrm{BMI} \mathbf{k g} \cdot \mathrm{m}^{-2}$} \\
\hline \multicolumn{2}{|l|}{$<25$} & 39 (32-45) & $9.0(5.1-13)$ & $0.1(0.0-0.6)$ \\
\hline \multicolumn{2}{|l|}{$25-29.9$} & 55 (46-63) & $26(18-33)$ & $9.2(0.4-14)$ \\
\hline \multicolumn{2}{|l|}{$\geqslant 30$} & 84 (72-95) & $54(39-69)$ & $19(7.1-30)$ \\
\hline \multicolumn{2}{|l|}{$p$-value } & $<0.001$ & $<0.001$ & $<0.001$ \\
\hline \multicolumn{5}{|l|}{ Hypertension } \\
\hline \multicolumn{2}{|c|}{ No hypertension } & 44 (39-49) & $17(13-21)$ & $4.0(1.9-6.1)$ \\
\hline \multicolumn{2}{|c|}{ Hypertension } & 80 (70-90) & $39(26-51)$ & $14(4.8-22)$ \\
\hline \multicolumn{2}{|l|}{$p$-value } & $<0.001$ & $<0.001$ & 0.002 \\
\hline \multicolumn{5}{|l|}{ Snoring } \\
\hline \multicolumn{2}{|l|}{ Nonsnorers } & $46(40-51)$ & $18(14-22)$ & $6.1(3.5-8.7)$ \\
\hline \multicolumn{2}{|l|}{ Snorers } & $66(53-79)$ & $34(21-47)$ & $6.3(0.0-13)$ \\
\hline \multicolumn{2}{|l|}{ p-value } & 0.007 & 0.009 & 0.96 \\
\hline \multicolumn{5}{|l|}{ Sleep apnoea } \\
\hline \multicolumn{2}{|c|}{ None witnessed } & $46(41-52)$ & $17(13-21)$ & $3.8(1.8-5.6)$ \\
\hline \multicolumn{2}{|c|}{ Witnessed } & $65(46-84)$ & $40(21-59)$ & $12(0.0-25)$ \\
\hline \multicolumn{2}{|l|}{ p-value } & 0.053 & 0.002 & 0.036 \\
\hline \multicolumn{5}{|l|}{ ESS } \\
\hline \multicolumn{2}{|l|}{$<10$} & $48(42-54)$ & $20(15-25)$ & $6.2(3.2-9.2)$ \\
\hline$\geqslant 10$ & & $48(39-57)$ & $18(11-25)$ & $2.7(0.0-5.4)$ \\
\hline p-value & & 0.99 & 0.64 & 0.13 \\
\hline Smoking & & & & \\
\hline Nonsmokers & & $48(42-53)$ & $19(15-24)$ & $5.0(2.5-7.3)$ \\
\hline Smokers & & $51(38-64)$ & $20(9.6-30)$ & $5.3(0.0-11)$ \\
\hline $\mathrm{p}$-value & & 0.62 & 0.93 & 0.88 \\
\hline Alcohol depe & ndent & & & \\
\hline No & & $48(43-53)$ & 19 (15-23) & $5.2(2.9-7.5)$ \\
\hline Yes & & 46 (23-69) & $24(4.0-43)$ & $1.8(0.0-8.0)$ \\
\hline$p$-value & & 0.82 & 0.57 & 0.49 \\
\hline
\end{tabular}

Data are presented as weighted mean $(95 \% \mathrm{Cl})$, unless otherwise stated. BMI: body mass index; ESS: Epworth Sleepiness Scale.

\section{RESULTS}

Polysomnographic recordings were made in all 400 females. Six females underwent a second polysomnography due to poor quality in the first recordings. The final analysis included 399 females, as one was excluded because she did not fall asleep during the recording. We had no minimal total sleep time for inclusion. The shortest total sleep time was $155 \mathrm{~min}$ and the longest was $569 \mathrm{~min}$. The weighted mean characteristics of the included females are given in table 1, while the weighted sleep characteristics are given in table 2 .

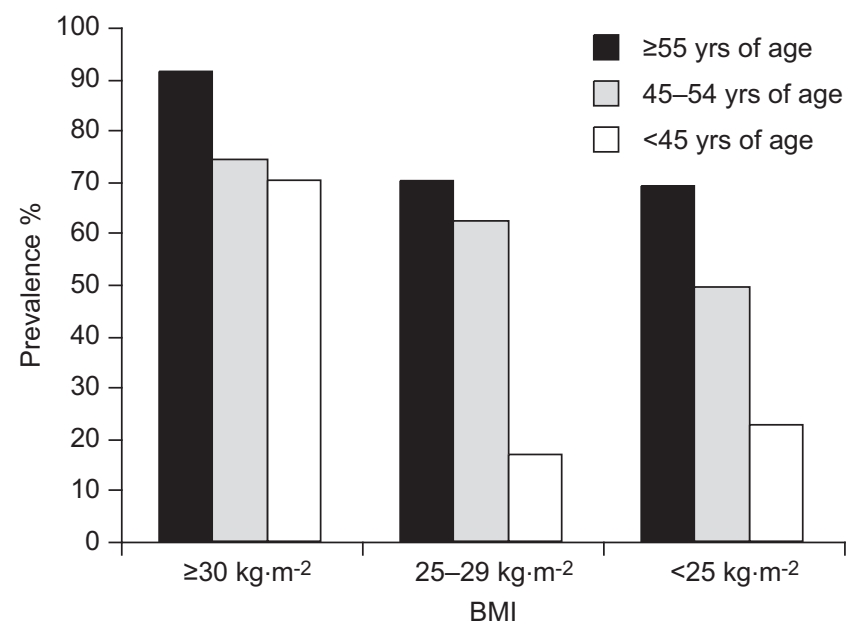

FIGURE 1. Prevalence (\%) of sleep apnoea (apnoea/hypopnoea index $\geqslant 5$ ) in females in relation to age and body mass index (BMI).

The weighted mean obstructive AHI among females aged 2070 yrs was 8.9 (95\% CI 7.8-10) events $\cdot h^{-1}$ and 50\% (95\% CI 45$55 \%$ ) of females had OSA with an AHI of $\geqslant 5$. Overweight, age, hypertension, witnessed apnoea and snoring were related to sleep apnoea, while daytime sleepiness, alcohol dependence and smoking were not (table 3).

OSA was found in $24 \%$ (95\% CI $45-55 \%$ ) of females aged 20 $44 \mathrm{yrs}$, in 56\% (95\% CI 47-65\%) of females aged 45-54 yrs and in $75 \%(95 \%$ CI $67-82 \%)$ of females aged $55-70$ yrs (table 3$)$. In the whole population, sleep apnoea occurred in $80 \%(95 \% \mathrm{CI}$ $70-90 \%$ ) of females with hypertension and in $84 \%$ (95\% CI $72-$ $95 \%$ ) of obese females with a BMI above $30 \mathrm{~kg} \cdot \mathrm{m}^{-2}$. The frequency of sleep apnoea in the population in relation to age and $\mathrm{BMI}$ is given in figures 1 and 2 .

Severe sleep apnoea $(\mathrm{AHI} \geqslant 30)$ occurred in $0.1 \%$ (95\% CI 0.0 $0.6 \%$ ) of females aged $20-45 \mathrm{yrs}$, in $4.6 \%$ (95\% CI $0.8-8.5 \%$ ) of females aged $45-54 \mathrm{yrs}$ and in $14 \%$ (95\% CI $8.1-21 \%$ ) of females aged $55-70$ yrs. As many as $31 \%$ (95\% CI $12-50 \%$ ) of obese females aged 55-70 yrs had severe sleep apnoea.

Hypertension and daytime sleepiness were observed as two different phenotypes in females with sleep apnoea. Hypertension occurred in $25 \%$ and daytime sleepiness in $34 \%$ of females with OSA (AHI $\geqslant 5$ ). Only $3.6 \%$ of females with sleep apnoea had the combination of both daytime sleepiness and hypertension. OSA was independently related to hypertension among females without sleepiness with an OR of 3.7 (95\% CI 1.3-10) after adjustments for age, BMI, alcohol dependence and smoking (table 4). The frequency of hypertension increased with the severity of sleep apnoea in univariate analysis (fig. 3 and table 4), but not in multivariate analysis because of the interaction with BMI.

Snoring was associated with sleep apnoea (table 3). Sleep apnoea was, however, more frequently observed than habitual snoring, and as many as $46 \%$ (95\% CI $50-51 \%$ ) of females who did not report that they snored habitually had an AHI of $\geqslant 5$ (table 3 ).

Central sleep apnoea with a central AHI of $\geqslant 5$ occurred in $1.3 \%$ (95\% CI $0.2-2.4 \%$ ) of females. All the females with central sleep apnoea also had OSA. 


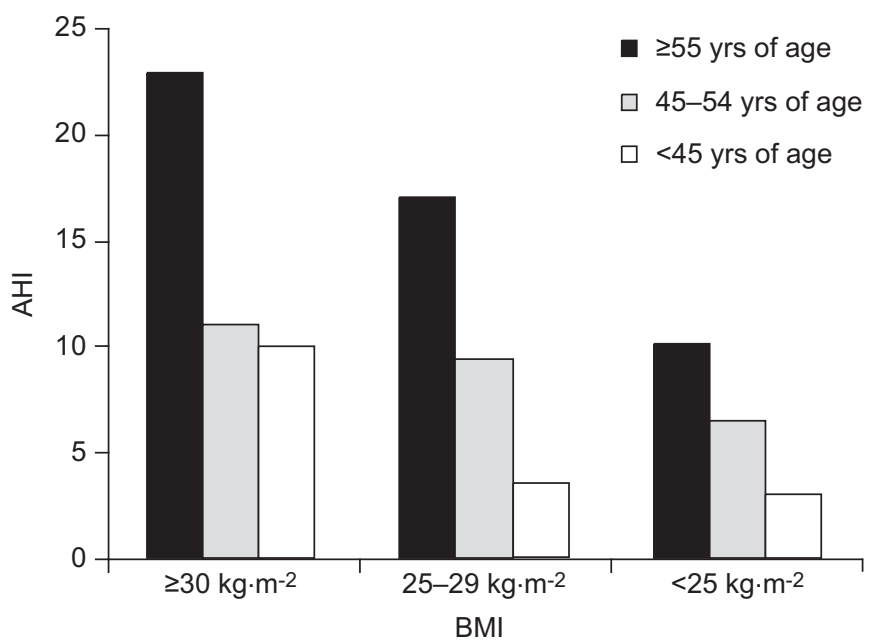

FIGURE 2. Mean number of apnoeas and hypopnoeas per hour of sleep (apnoea/hypopnoea index; AHI) in females in relation to age and body mass index (BMI).

\section{DISCUSSION}

Unexpectedly, we found that as many as 50\% (95\% CI 45-55\%) of females in the general population fulfilled the criterion for sleep apnoea, with $\geqslant 5$ apnoeas and hypopnoeas per hour of sleep. Sleep apnoea occurred in 80\% (95\% CI 70-90\%) of females with hypertension and in $84 \%$ (95\% CI 72-95\%) of obese females. Severe sleep apnoea occurred in 14\% (95\% CI 8.1-21\%) of females aged $55-70$ yrs and in 31\% (95\% CI 12-50\%) of obese females aged 55-70 yrs. Sleep apnoea was related to age, obesity, hypertension, witnessed apnoea and snoring, but not to daytime sleepiness, alcohol dependence or smoking.

Daytime sleepiness and hypertension were observed as two different phenotypes of sleep apnoea. $34 \%$ of the present females with sleep apnoea were sleepy during the daytime, $25 \%$ had hypertension and only $3.6 \%$ suffered from the combination of both sleepiness and hypertension. Hypertension was independently related to OSA in females without daytime sleepiness.

Snoring and daytime sleepiness are the main reasons for males and females to seek medical attention for sleep apnoea. However, daytime sleepiness was not related to sleep apnoea in the present females and most females with sleep apnoea did not snore. Habitual snoring occurred in $7.6 \%$ of the present sample. It was associated with sleep apnoea, but habitual snoring was not as common as sleep apnoea. A previous study also reports that severe sleep apnoea occurs in nonsnoring females [15].

The sleep apnoea syndrome is defined as an AHI of $\geqslant 5$, in combination with excessive daytime sleepiness $[2,20]$. Daytime sleepiness is also a main indication for the treatment of sleep apnoea $[10,11,13]$. Previous epidemiological studies report that the majority of females with an AHI of $\geqslant 5$ do not complain of daytime sleepiness [15, 17-19], and that sleep apnoea is not related to excessive daytime sleepiness [17-19]. In 1993, using positive answers to three questions on hypersomnolence, YounG et al. [15] reported that 23\% of females with sleep apnoea were tired during the daytime. Using the ESS, we also found that the vast majority of females

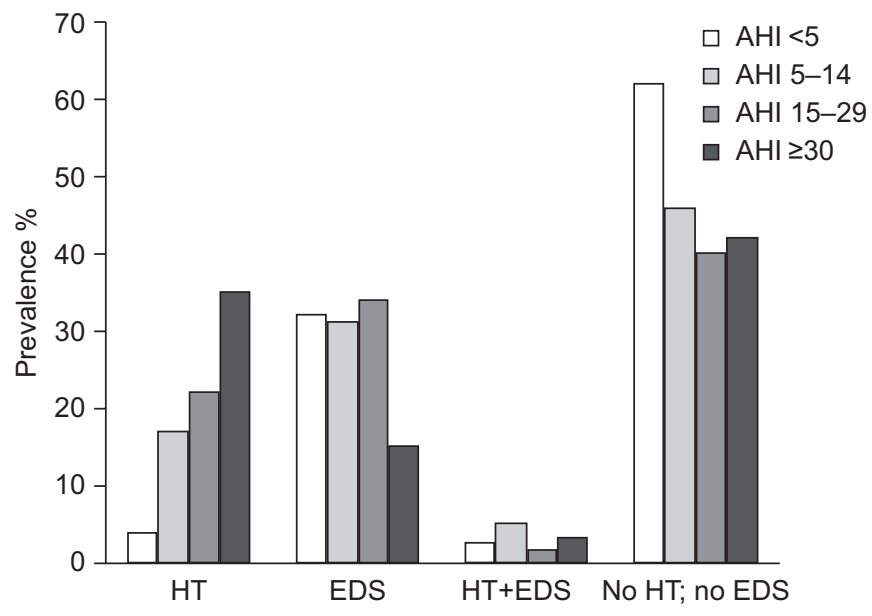

FIGURE 3. Prevalence (\%) of hypertension (HT) and excessive daytime sleepiness (EDS) with regard to the apnoea/hypopnoea index (AHI).

with sleep apnoea were not tired during the daytime. Daytime sleepiness was not related to sleep apnoea in the present females, in mild, moderate or severe sleep apnoea. Instead, obesity, hypertension and older age were key signs of sleep apnoea in females. It is possible that sleep apnoea has not been observed as a public health problem in females, as they have other signs of sleep apnoea than males.

In 1993, Young et al. [15] reported that 9\% of state-employed females had an AHI $\geqslant 5$. DURAN et al. [17] from Spain, KIM et al. [18] from Korea and Ip et al. [19] from China report a frequency of sleep apnoea in females of $4 \%$ to $28 \%$. These studies used a two-phase investigation system with a postal questionnaire or telephone enquiry to a large group of females and polysomnograms to a limited group. We used modern equipment, new diagnostic criteria and included females from the population, and observed a much higher prevalence of sleep apnoea in females than that reported previously. Air flow was measured in the present study using both a nasal pressure cannula and an oronasal thermistor [2, 20]. Hypopnoeas have previously been defined in different ways and a reduction in air flow of $50 \%$, followed by a desaturation of $4 \%$, has often been used. The new recommendations, which were used in the present study, include desaturations of at least 3\% instead of $4 \%$ after a reduction in air flow of 50\% $[2,20]$. Different inclusion criteria and different populations at another time point can possibly also explain some of the differences.

It is known that the prevalence of sleep apnoea increases with age, but the relevance of sleep apnoea defined as an AHI of $\geqslant 5$ without symptoms in older ages is uncertain. The exact cut-off for the AHI as a risk factor for cardiovascular disease in older ages is also unknown. However, recent long-term follow-up data from the Wisconsin Sleep Cohort and the Sleep Heart Health Study observed that severe sleep apnoea with an AHI of $\geqslant 30$ is associated with a marked increase in mortality in the population [28-30]. YouNG et al. [28] followed 1,522 males and females in Wisconsin during a period of 18 yrs and observed a tripling in the risk of all-cause mortality adjusted for age, sex, $\mathrm{BMI}$ and other factors among subjects with severe sleep apnoea 


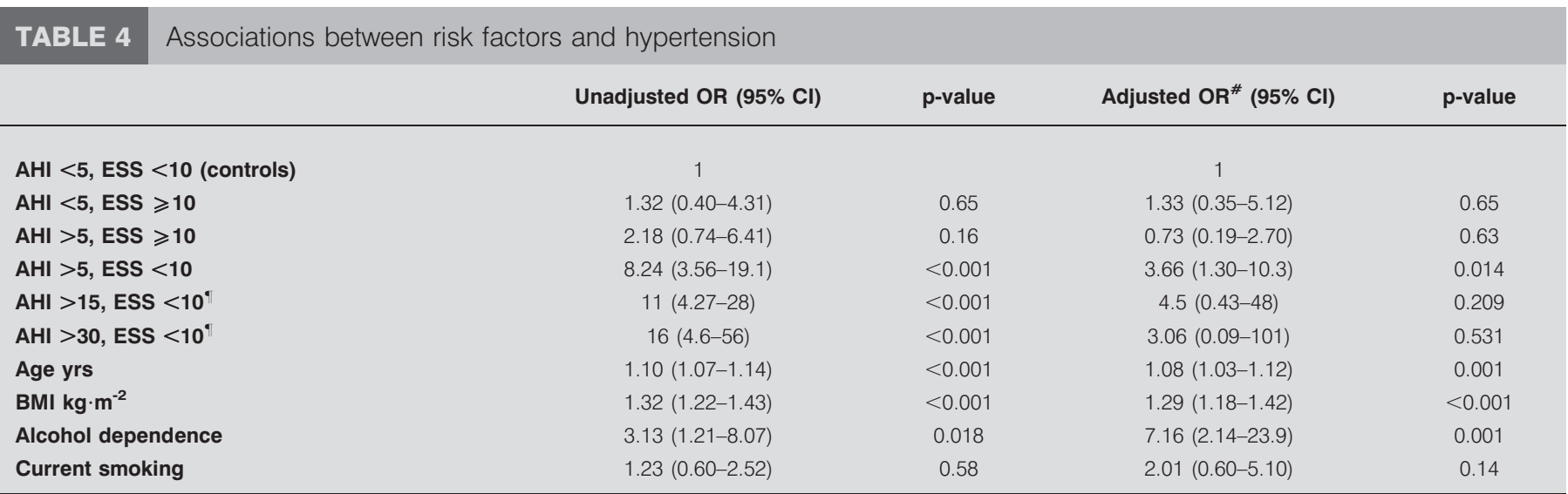

AHI: apnoea/hypopnoea index; ESS: Epworth Sleepiness Scale; BMI: body mass index. "\#: adjusted for all the factors in the table; ": analysed separately with AHI <5, ESS $<10$ as controls.

versus no sleep apnoea. PUNJABI et al. [29] followed 6,441 males and females for 8.2 yrs in the Sleep Heart Health Study. Males with severe sleep apnoea aged $<70$ yrs had double the risk of death. Corresponding females had a similar odds ratio of 1.76 that did not reach significance, which could be due to power problems, as fewer females with severe sleep apnoea and fewer females than males died during the follow-up.

We found that about $4.6 \%$ of females aged $45-54$ yrs and as many as $14 \%$ of females aged $55-70$ yrs and $31 \%$ of obese females aged 55-70 yrs have severe sleep apnoea with an AHI of $\geqslant 30$. It is necessary to identify these females with unrecognised severe sleep apnoea as they probably run an increased risk of early death, without adequate treatment for OSA. We used different polysomnographic technology and different scoring criteria compared with the Wisconsin Sleep Cohort and the Sleep Heart Health Study, and found a higher prevalence of severe sleep apnoea and it is therefore not certain that the present females with severe sleep apnoea share the same risk of death [28, 29].

Smoking and alcohol consumption are often considered as risk factors for sleep apnoea despite limited evidence, especially in females. It is well known that alcohol consumption before going to bed worsens sleep apnoea in males [31], and that smoking is related to snoring in males and females [22, 32]. Drinking $>23 \mathrm{~g}$ of alcohol per day was related to oxygen desaturations and snoring in a study of Japanese females [33]. Smoking was related to sleep apnoea and alcohol to sleep apnoea in males but not in females in the Wisconsin Sleep Cohort [34, 35]. However, many epidemiological studies do not report any association between OSA and smoking or alcohol [18, 19, 36, 37]. In support of these studies, we did not find any association between OSA and smoking or drinking alcohol in females.

The limitations of the present study include data on snoring as females may snore despite a negative answer because they are single, have problems with awake bed-partners' observations or lack a "don't know answer" to the question on snoring. We used modern diagnostic equipment, including nasal airflow, new definitions for apnoea and hypopnoea and performed polysomnography at home, which is a strength but also a limitation when comparing the present results with older studies. Daytime sleepiness was scored using the ESS, as it is frequently used. There are, however, many limitations, including the subjective nature, and it might be less applicable in females as it includes questions on car driving and females drive less than males [38]. There is a need for the identification of specific symptoms of sleep apnoea in females. We investigated females only and we cannot compare females with males, which is another limitation in the present study, especially when it comes to compare our results with previous epidemiological studies.

In conclusion, OSA occurs in 50\% of females aged $20-70$ yrs. $20 \%$ of females have moderate and $6 \%$ have severe sleep apnoea. Sleep apnoea in females is related to age, obesity and hypertension but not to daytime sleepiness. When searching for sleep apnoea in females, those with hypertension or obesity should be investigated.

\section{SUPPORT STATEMENT}

This study was funded by grants from the Swedish Heart Lung Foundation.

\section{STATEMENT OF INTEREST}

None declared.

\section{REFERENCES}

1 Franklin KA, Nilsson JB, Sahlin C, et al. Sleep apnoea and nocturnal angina. Lancet 1995; 345: 1085-1087.

2 American Academy of Sleep Medicine Task Force. Sleep-related breathing disorders in adults: recommendations for syndrome definition and measurement techniques in clinical research. The Report of an American Academy of Sleep Medicine Task Force. Sleep 1999; 22: 667-689.

3 Peppard PE, Young T, Palta M, et al. Prospective study of the association between sleep-disordered breathing and hypertension. N Engl J Med 2000; 342: 1378-1384.

4 Malhotra A, White DP. Obstructive sleep apnoea. Lancet 2002; 360: 237-245.

5 Marin JM, Carrizo SJ, Vicente E, et al. Long-term cardiovascular outcomes in men with obstructive sleep apnoea-hypopnoea with 
or without treatment with continuous positive airway pressure: an observational study. Lancet 2005; 365: 1046-1053.

6 Yaggi HK, Concato J, Kernan WN, et al. Obstructive sleep apnea as a risk factor for stroke and death. N Engl J Med 2005; 353: 2034-2041.

7 Valham F, Mooe T, Rabben T, Stenlund H, et al. Increased risk of stroke in patients with coronary artery disease and sleep apnea: a 10-year follow-up. Circulation 2008; 118: 955-960.

8 Sahlin C, Sandberg O, Gustafson Y, et al. Obstructive sleep apnea is a risk factor for death in patients with stroke: a 10-year followup. Arch Intern Med 2008; 168: 297-301.

9 Bonsignore MR, Zito A. Metabolic effects of the obstructive sleep apnea syndrome and cardiovascular risk. Arch Physiol Biochem 2008; 114: 255-260.

10 Sullivan CE, Issa FG, Berthon-Jones $\mathrm{M}$, et al. Reversal of obstructive sleep apnoea by continuous positive airway pressure applied through the nares. Lancet 1981; 1: 862-865.

11 Giles TL, Lasserson TJ, Smith BJ, et al. Continuous positive airways pressure for obstructive sleep apnoea in adults. Cochrane Database Syst Rev 2006; 1: CD001106.

12 Lim J, Lasserson TJ, Fleetham J, et al. Oral appliances for obstructive sleep apnoea. Cochrane Database Syst Rev 2006; 25: CD004435.

13 Diagnosis and treatment in obstructive sleep apnea syndromes. A systematic review by DACEHTA in Denmark, FinOHTA in Finland, NHSRC in Norway, Landspitali in Island and SBU in Sweden. Stockholm, SBU, 2007.

14 Barbe F, Duran-Cantolla J, Capote F, et al. Long-term effect of continuous positive airway pressure in hypertensive patients with sleep apnea. Am J Respir Crit Care Med, 181: 718-726.

15 Young T, Palta M, Dempsey J, et al. The occurrence of sleepdisordered breathing among middle-aged adults. N Engl J Med 1993; 328: 1230-1235.

16 Bixler EO, Vgontzas AN, Lin HM, et al. Prevalence of sleepdisordered breathing in women: effects of gender. Am J Respir Crit Care Med 2001; 163: 608-613.

17 Duran J, Esnaola S, Rubio R, et al. Obstructive sleep apneahypopnea and related clinical features in a population-based sample of subjects aged 30 to $70 \mathrm{yr}$. Am J Respir Crit Care Med 2001; 163: 685-689.

18 Kim J, In K, You S, et al. Prevalence of sleep-disordered breathing in middle-aged Korean men and women. Am J Respir Crit Care Med 2004; 170: 1108-1113.

19 Ip MS, Lam B, Tang LC, et al. A community study of sleepdisordered breathing in middle-aged Chinese women in Hong Kong: prevalence and gender differences. Chest 2004; 125: 127-134.

20 Iber C, Ancoli-Israel S, Chesson A, et al. eds. The AASM Manual for the Scoring of Sleep and Associated Events: Rules, Terminology and technical specifications. 1st Edn. Westchester, Illinois, American Academy of Sleep Medicine, 2007.

21 Theorell-Haglow J, Lindberg E, Janson C. What are the important risk factors for daytime sleepiness and fatigue in women? Sleep 2006; 29: 751-757.
22 Svensson M, Lindberg E, Naessen T, et al. Risk factors associated with snoring in women with special emphasis on body mass index: a population-based study. Chest 2006; 129: 933-941.

23 Sahlin C, Franklin KA, Stenlund H, et al. Sleep in women: normal values for sleep stages and position and the effect of age, obesity, sleep apnea, smoking, alcohol and hypertension. Sleep Med 2009; 10: $1025-1030$.

24 Theorell-Haglow J, Berne C, Janson C, et al. Associations between short sleep duration and central obesity in women. Sleep 2010; 33: 593-598.

25 Rechtschaffen A, Kales A. A manual of standardized terminology, techniques and scoring system for sleep stages of human subjects. Los Angeles, Barin Information Service/Brain Research Institute, 1968.

26 Johns MW. Sleepiness in different situations measured by the Epworth Sleepiness Scale. Sleep 1994; 17: 703-710.

27 Ewing JA. Detecting alcoholism. The CAGE questionnaire. JAMA 1984; 252: 1905-1907.

28 Young T, Finn L, Peppard PE, et al. Sleep disordered breathing and mortality: eighteen-year follow-up of the Wisconsin sleep cohort. Sleep 2008; 31: 1071-1078.

29 Punjabi NM, Caffo BS, Goodwin JL, et al. Sleep-disordered breathing and mortality: a prospective cohort study. PLoS Med 2009; 6: e1000132.

30 Pack AI, Platt AB, Pien GW. Does untreated obstructive sleep apnea lead to death? A commentary on Young et al. Sleep 2008; 31: 1071-8 and Marshall et al. Sleep 2008; 31: 1079-85. Sleep 2008; 31: 1067-1068.

31 Scanlan MF, Roebuck T, Little PJ, et al. Effect of moderate alcohol upon obstructive sleep apnoea. Eur Respir J 2000; 16: 909-913.

32 Franklin KA, Gislason T, Omenaas E, et al. The influence of active and passive smoking on habitual snoring. Am J Respir Crit Care Med 2004; 170: 799-803.

33 Cui R, Tanigawa T, Sakurai S, et al. Associations between alcohol consumption and sleep-disordered breathing among Japanese women. Respir Med 105: 796-800.

34 Peppard PE, Austin D, Brown RL. Association of alcohol consumption and sleep disordered breathing in men and women. J Clin Sleep Med 2007; 3: 265-270.

35 Wetter DW, Young TB, Bidwell TR, et al. Smoking as a risk factor for sleep-disordered breathing. Arch Intern Med 1994; 154: 2219-2224.

36 Newman $\mathrm{AB}$, Nieto FJ, Guidry $\mathrm{U}$, et al. Relation of sleepdisordered breathing to cardiovascular disease risk factors: the Sleep Heart Health Study. Am J Epidemiol 2001; 154: 50-59.

37 Tishler PV, Larkin EK, Schluchter MD, et al. Incidence of sleepdisordered breathing in an urban adult population: the relative importance of risk factors in the development of sleep-disordered breathing. Jama 2003; 289: 2230-2237.

38 Valham F, Eriksson M, Stegmayr B, et al. Snoring men with daytime sleepiness drive more than others: a population-based study. Sleep Med 2009; 10: 1012-1015. 